\title{
'Interpolating' differential reductions of multidimensional integrable hierarchies
}

\author{
L.V. Bogdanov*
}

November 13, 2018

\begin{abstract}
We transfer the scheme of constructing differential reductions, developed recently for the case of the Manakov-Santini hierarchy, to the general multidimensional case. We consider in more detail the fourdimensional case, connected with the second heavenly equation and its generalization proposed by Dunajski. We give a characterization of differential reductions in terms of the Lax-Sato equations as well as in the framework of the dressing method based on nonlinear Riemann-Hilbert problem.
\end{abstract}

\section{Introduction}

Recently [1 we have constructed a class of reductions of the hierarchy associated with the system introduced by Manakov and Santini [2] (see also [3], [4]),

$$
\begin{aligned}
& u_{x t}=u_{y y}+\left(u u_{x}\right)_{x}+v_{x} u_{x y}-u_{x x} v_{y} \\
& v_{x t}=v_{y y}+u v_{x x}+v_{x} v_{x y}-v_{x x} v_{y}
\end{aligned}
$$

In [5] it was shown that a simple differential reduction

$$
\alpha u=v_{x}
$$

(where $\alpha$ is a constant) of the Manakov-Santini system (10) corresponds to the interpolating system, which was introduced in [5] as "the most general symmetry reduction of the second heavenly equation by a conformal Killing

${ }^{*}$ L.D. Landau ITP RAS, 142432 Chernogolovka, Moscow region, Russia; e-mail leonid@landau.ac.ru 
vector with a null self-dual derivative". The term 'interpolating' is connected with the fact that the system (1) with the reduction (2) 'interpolates' between the dKP equation, arising as $\alpha \rightarrow 0$, and the system considered in [6] (see also [7, 8, 9]), arising at $\alpha \rightarrow \infty$. Using Lax-Sato formulation of the Manakov-Santini hierarchy [10, 11, in [1] we introduced a class of reductions, such that the zero order reduction of this class corresponds to the dKP hierarchy, and the first order reduction gives a hierarchy associated with the interpolating system. We presented the generating equation and the Lax-Sato form of a reduced hierarchy for the interpolating system and also for the reduction of an arbitrary order. Reduction of every order $k$ contains a parameter $\alpha$ and is in some sence interpolating, providing the dKP hierarchy for $\alpha \rightarrow 0$ and Gelfand-Dikii reduction of the order $k$ for the Manakov-Santini hierarchy at $\alpha \rightarrow \infty$. It is important to note that Gelfand-Dikii reduction for the Manakov-Santini hierarchy does't imply the stationarity with respect to some higher time. A characterization of the class of reductions in terms of the dressing data was given.

In this work we transfer the construction developed in [1] to the case of general (N+2)-dimensional hierarchy [10, 11]. The Manakov-Santini hierarchy corresponds to $N=1$, this case is nongeneric, and there are some new features in the general case. We pay special attention to the case $N=2$, which contains Plebański second heavenly equation [12] and its generalization proposed by Dunajski [13].

It is interesting to note a deep structural similarity of our construction with the picture of differential reductions in the standard (dispersionful) integrable case [14, 15, 16, 17, though the technical tools are essentially different.

\section{The hierarchy}

The Manakov-Santini hierarchy represents a special case corresponding to $\mathrm{N}=1$ of the general $(\mathrm{N}+2)$-dimensional hierarchy defined by the generating relation

$$
\left(J_{0}^{-1} \mathrm{~d} \Psi^{0} \wedge \mathrm{d} \Psi^{1} \wedge \cdots \wedge \mathrm{d} \Psi^{N}\right)_{-}=0
$$


where $\Psi^{0}, \ldots, \Psi^{N}$ are the series

$$
\begin{aligned}
\Psi^{0} & =\lambda+\sum_{n=1}^{\infty} \Psi_{n}^{0}\left(\mathbf{t}^{1}, \ldots, \mathbf{t}^{N}\right) l^{-n}, \\
\Psi^{k} & =\sum_{n=0}^{\infty} t_{n}^{k}\left(\Psi^{0}\right)^{n}+\sum_{n=1}^{\infty} \Psi_{n}^{k}\left(\mathbf{t}^{1}, \ldots, \mathbf{t}^{N}\right)\left(\Psi^{0}\right)^{-n},
\end{aligned}
$$

$1 \leqslant k \leqslant N, \mathbf{t}^{k}=\left(t_{0}^{k}, \ldots, t_{n}^{k}, \ldots\right),(\cdots)_{-}$is a projection to negative powers of $\lambda, J_{0}$ is a determinant of Jacobian matrix $J$,

$$
J_{0}=\operatorname{det} J, \quad J_{i j}=\partial_{i} \Psi^{j}, \quad 0 \leqslant i, j \leqslant N,
$$

where $\partial_{0}=\frac{\partial}{\partial \lambda}, \partial_{k}=\frac{\partial}{\partial x^{k}}(1 \leqslant k \leqslant N), x^{k}=t_{0}^{k}$.

Generating relation (3) is equivalent to the set of Lax-Sato equations

$$
\left.\partial_{n}^{k} \boldsymbol{\Psi}=\sum_{i=0}^{N}\left(\left(J^{-1}\right)_{k i}\left(\Psi^{0}\right)^{n}\right)\right)_{+} \partial_{i} \boldsymbol{\Psi}, \quad 0 \leqslant n \leqslant \infty, 1 \leqslant k \leqslant N,
$$

where $\boldsymbol{\Psi}=\left(\Psi^{0}, \ldots, \Psi^{N}\right),(\cdots)_{+}$is a projection to nonnegative powers of $\lambda$. First flows of the hierarchy read

$$
\partial_{1}^{k} \boldsymbol{\Psi}=\left(\lambda \partial_{k}-\sum_{p=1}^{N}\left(\partial_{k} u_{p}\right) \partial_{p}-\left(\partial_{k} u_{0}\right) \partial_{\lambda}\right) \boldsymbol{\Psi}, \quad 0<k \leqslant N,
$$

where $u_{0}=\Psi_{1}^{0}, u_{k}=\Psi_{1}^{k}, 1 \leqslant k \leqslant N$. A compatibility condition for any pair of linear equations (e.g., with $\partial_{1}^{k}$ and $\partial_{1}^{q}, k \neq q$ ) implies closed nonlinear $(\mathrm{N}+2)$-dimensional system of PDEs for the set of functions $u_{k}, u_{0}$, which can be written in the form

$$
\begin{aligned}
& \partial_{1}^{k} \partial_{q} \hat{u}-\partial_{1}^{q} \partial_{k} \hat{u}+\left[\partial_{k} \hat{u}, \partial_{q} \hat{u}\right]=\left(\partial_{k} u_{0}\right) \partial_{q}-\left(\partial_{q} u_{0}\right) \partial_{k}, \\
& \partial_{1}^{k} \partial_{q} u_{0}-\partial_{1}^{q} \partial_{k} u_{0}+\left(\partial_{k} \hat{u}\right) \partial_{q} u_{0}-\left(\partial_{q} \hat{u}\right) \partial_{k} u_{0}=0,
\end{aligned}
$$

where $\hat{u}$ is a vector field, $\hat{u}=\sum_{p=1}^{N} u_{k} \partial_{k}$.

\section{3 'Intepolating' differential reductions}

Lax-Sato equations of the hierarchy (7)

$$
\left.\partial_{n}^{k} \boldsymbol{\Psi}=\hat{V}_{n}^{k} \mathbf{\Psi}, \quad \hat{V}_{n}^{k}=\sum_{i=0}^{N} V_{n i}^{k} \partial_{i}, \quad V_{n i}^{k}=\left(\left(J_{0}^{-1}\right)_{k i}\left(\Psi^{0}\right)^{n}\right)\right)_{+}
$$


imply linear equations for the Jacobian (6)

$$
\partial_{n}^{k} J_{0}=\sum_{i=0}^{N} \partial_{i}\left(V_{n i}^{k} J_{0}\right)
$$

These equations may be considered as formally adjoint to linear equations (10), where we define adjoint operator for $u_{i} \partial_{i}$ as $-\partial_{i} u_{i}$. It is interesting to note that the adjoint equations coincide with equations (10) only in the case of divergence-free vector fields.

Equivalently, equations (11) can be written as nonhomogeneous linear equations for the logarithm of Jacobian,

$$
\partial_{n}^{k} \ln J_{0}=\hat{V}_{n}^{k} \ln J_{0}+\operatorname{div} \hat{V}_{n}^{k}, \quad \operatorname{div} \hat{V}_{n}^{k}=\sum_{i=0}^{N} \partial_{i} V_{n i}^{k} .
$$

The coefficients of these equations are polynomial in $\lambda$, the function $\left(\ln J_{0}-\right.$ $\left.\alpha\left(\Psi^{0}\right)^{k}\right)$ is a solution of the equations, thus the condition

$$
\left(\ln J_{0}-\alpha\left(\Psi^{0}\right)^{k}\right)_{-}=0 .
$$

defines a reduction of the hierarchy (this condition is preserved by the dynamics). Similar to the case of the Manakov-Santini hierarchy, this condition is characterized by the existence of a polynomial solution of equations (12).

Proposition 1 The existence of a polynomial solution of the form

$$
f=-\alpha l^{k}+\sum_{0}^{i=k-2} f_{i}(\mathbf{t}) l^{i}
$$

(where coefficients $f_{i}$ don't contain constants) of equations (12),

$$
\partial_{n}^{k} f=\hat{V}_{n}^{k} f+\operatorname{div} \hat{V}_{n}^{k}
$$

is equivalent to the reduction condition (13).

Proof The proof is completely analogous to the proof of similar statement for the Manakov-Santini hierarchy, given in [1]. First, the reduction condition (13) directly implies that $f=\left(\ln J_{0}-\alpha\left(\Psi^{0}\right)^{k}\right)$ is a polynomial solution of equations (12) of required form, thus the existence of a polynomial solution is necessary. 
To prove that it is sufficient, we note that $F=\ln J_{0}-f$ solves homogeneous equations (12) (equations (10)). Let us expand $l$ into the powers of $\Psi^{0}$, reverting the series (4), and represent $F$ in the form

$$
F=\alpha\left(\Psi^{0}\right)^{k}+\sum_{-\infty}^{i=k-2} F_{i}(\mathbf{t})\left(\Psi^{0}\right)^{i}
$$

It is easy to check that $F$ solves homogeneous equations (12) iff all the coefficients $F_{i}(\mathbf{t})$ are constants. Suggesting that the coefficients $f_{i}$ of the polynomial $f$ don't contain constants, we come to the conclusion that $\ln J_{0}-$ $\alpha\left(\Psi^{0}\right)^{k}=f$, thus $\left(\ln J_{0}-\alpha\left(\Psi^{0}\right)^{k}\right)_{-}=0$

Another equivalent formulation of the reduction can be presented in terms of the generating relation (3). Reduction condition (13) implies that

$$
J_{0}=\exp \left(\alpha\left(\Psi^{0}\right)_{-}^{k}\right)
$$

and it is easy to prove the following statement:

Proposition 2 The reduced hierarchy defined by the generating relation (3) together with the reduction condition (13) is equivalent to the generating relation

$$
\left(\exp \left(-\alpha\left(\Psi^{0}\right)^{k}\right) \mathrm{d} \Psi^{0} \wedge \mathrm{d} \Psi^{1} \wedge \cdots \wedge \mathrm{d} \Psi^{N}\right)_{-}=0
$$

To calculate the reduction in terms of equations (9), it is convenient to start from nonhomogeneous linear equations (12), coresponding to the first flows of the hierarchy (8), which read

$$
\partial_{1}^{n} f=\left(\lambda \partial_{n}-\sum_{p=1}^{N}\left(\partial_{n} u_{p}\right) \partial_{p}-\left(\partial_{n} u_{0}\right) \partial_{\lambda}\right) f-\partial_{n} \sum_{p=1}^{N} \partial_{p} u_{p}, 1 \leqslant n \leqslant N .
$$

Substituting $f$ of the form (14) to these equations, we obtain $k-1$ equations for $k$ coefficients $f_{i}$, which determine $f_{i}$ through the functions $u_{0}, u_{n}$ and define a differential reduction.

\section{$k=0$. Divergence-free vector fields}

For $k=0$ the reduction condition (13), taking into account that the expansion of $J_{0}$ is of the form $J_{0}=1+J_{0}^{1} l^{-1}+\ldots\left(\right.$ so $\left.\left(\ln J_{0}\right)_{+}=0\right)$, implies 
that $J_{0}=1$. Thus nonhomogeneous linear equations (12) possess a solution equal to zero, and substituting it to the equations, we get

$$
\operatorname{div} \hat{V}_{n}^{k}=0
$$

so the vector fields in the Lax-Sato equations (10) are divergence-free, and the flows of the hierarchy are volume-preserving. Generating equation (3) for the reduced hierarchy reads

$$
\left(\mathrm{d} \Psi^{0} \wedge \mathrm{d} \Psi^{1} \wedge \cdots \wedge \mathrm{d} \Psi^{N}\right)_{-}=0 .
$$

On the other hand, volume-preserving reduction can be obtained from the reduction (13) with arbitrary $k$ in the limit $\alpha \rightarrow 0$. Thus the reduction (13) with arbitrary $k$ is an 'interpolating' reduction between the volumepreserving hierarchy and the hierarchy, characterized by the existence of polynomial solution of Lax-Sato equations (7) (Gelfand-Dikii reduction).

To calculate the reduction in terms of equations (9), we substitute zero solution to equations (16) and obtain a condition

$$
\operatorname{div} \hat{u}:=\sum_{p=1}^{N} \partial_{p} u_{p}=0 .
$$

Thus equations (9) reduce to divergence-free vector fields $\hat{u}$.

\section{The reduction for $\mathrm{k}=1$}

For $k=1$ the reduction condition (13) reads

$$
\left(\ln J_{0}-\alpha \Psi^{0}\right)_{-}=0,
$$

thus

$$
\ln J_{0}-\alpha \Psi^{0}=-\left(\alpha \Psi^{0}\right)_{+},
$$

and for the Jacobian we obtain

$$
J_{0}=\exp \alpha\left(\Psi^{0}-\lambda\right) .
$$

Generating equation for the reduced hierarchy is

$$
\left(\exp \left(-\alpha \Psi^{0}\right) \mathrm{d} \Psi^{0} \wedge \mathrm{d} \Psi^{1} \wedge \cdots \wedge \mathrm{d} \Psi^{N}\right)_{-}=0 .
$$


The reduction implies the existence of the solution $-\alpha \lambda$ of nonhomogeneous linear equations (12) and leads to the relations

$$
\operatorname{div} \hat{V}_{n}^{k}=\alpha V_{n 0}^{k}
$$

Using these relations for the first flows (16), we calculate the reduction condition for equations (9),

$$
\operatorname{div} \hat{u}:=\sum_{p=1}^{N} \partial_{p} u_{p}=\alpha u_{0} .
$$

Using this relation, it is possible to exclude the function $u_{0}$ from the system (9) and obtain the reduced system in explicit form,

$$
\partial_{1}^{k} \partial_{q} \hat{u}-\partial_{1}^{q} \partial_{k} \hat{u}+\left[\partial_{k} \hat{u}, \partial_{q} \hat{u}\right]=\alpha^{-1}\left(\left(\partial_{k} \operatorname{div} \hat{u}\right) \partial_{q}-\left(\partial_{q} \operatorname{div} \hat{u}\right) \partial_{k}\right) .
$$

The limit $\alpha \rightarrow 0$ of the reduced hierarchy corresponds to the volumepreserving case $(\mathrm{k}=0)$ described above, while the limit $\alpha \rightarrow \infty$ corresponds to the hierarchy characterized by the relation $\Psi^{0}=l[18,[10$. For this hierarchy vector fields of Lax-Sato equations (7) do not contain a derivative with respect to a spectral variable, and $u_{0}$ in equations (9) is equal to zero,

$$
\partial_{1}^{k} \partial_{q} \hat{u}-\partial_{1}^{q} \partial_{k} \hat{u}+\left[\partial_{k} \hat{u}, \partial_{q} \hat{u}\right]=0 .
$$

This hierarchy is a 'precursor' of hyper-Kahler hierarchies [19, 20], which correspond to Hamiltonian vector fiels both in Lax-Sato equations (7) and in equations (9). Thus the reduction (13) with $k=1$ is 'interpolating' between the volume-preserving hierarchy, connected with the system (9) for divergence-free vector fields $\hat{u}$, and pre-hyper-Kahler hierarchy connected with the system (19).

$\mathrm{k}=\mathbf{2}$

For $k=2$ the reduction condition (13) reads

$$
\left(\ln J_{0}-\alpha\left(\Psi^{0}\right)^{2}\right)_{-}=0,
$$

thus

$$
\ln J_{0}-\alpha\left(\Psi^{0}\right)^{2}=-\alpha\left(\Psi^{0}\right)_{+}^{2},
$$

and for the Jacobian we obtain

$$
J_{0}=\exp \alpha\left(\left(\Psi^{0}\right)_{-}^{2}\right) .
$$


Generating equation for the reduced hierarchy is

$$
\left(\exp \left(-\alpha\left(\Psi^{0}\right)^{2}\right) \mathrm{d} \Psi^{0} \wedge \mathrm{d} \Psi^{1} \wedge \cdots \wedge \mathrm{d} \Psi^{N}\right)_{-}=0 .
$$

The reduction implies the existence of polynomial solution $f=-\alpha l^{2}+$ $f_{1}$ of nonhomogeneous linear equations (12). Substituting this solution to equations (16), we obtain

$$
\begin{aligned}
& \partial_{1}^{n} f_{1}=-\left(\partial_{n} \hat{u}\right) f_{1}-\partial_{n} \operatorname{div} \hat{u}, \\
& \partial_{n} f_{1}=-2 \alpha\left(\partial_{n} u_{0}\right),
\end{aligned}
$$

or, excluding $f_{1}$,

$$
\partial_{1}^{n} u_{0}+\left(\partial_{n} \hat{u}\right) u_{0}+\frac{1}{2 \alpha} \partial_{n} \operatorname{div} \hat{u}=0 .
$$

These relations represent a differential reduction for the system (9). It is not difficult to check that the substitution of these relations to the second equation of the system (9) satisfies it identically.

The limit $\alpha \rightarrow 0$ of the reduced hierarchy corresponds to the volumepreserving case $(\mathrm{k}=0)$, while the limit $\alpha \rightarrow \infty$ corresponds to the hierarchy characterized by the relation $\left(\Psi^{0}\right)_{-}^{2}=0$, or, equivalently, $\left(\Psi^{0}\right)^{2}=l^{2}+2 u_{0}$ (Gelfand-Dikii reduction).

$\mathbf{k}=\mathbf{3}$

For $k=3$ the reduction condition (13) reads

$$
\left(\ln J_{0}-\alpha\left(\Psi^{0}\right)^{3}\right)_{-}=0,
$$

and for the Jacobian we obtain

$$
J_{0}=\exp \alpha\left(\left(\Psi^{0}\right)_{-}^{3}\right) .
$$

Generating equation for the reduced hierarchy is

$$
\left(\exp \left(-\alpha\left(\Psi^{0}\right)^{3}\right) \mathrm{d} \Psi^{0} \wedge \mathrm{d} \Psi^{1} \wedge \cdots \wedge \mathrm{d} \Psi^{N}\right)_{-}=0 .
$$

The reduction implies the existence of polynomial solution $f=-\alpha l^{3}+f_{1} l+$ $f_{2}$ of nonhomogeneous linear equations (12). Substituting this solution to equations (16), we obtain

$$
\begin{aligned}
& \partial_{1}^{n} f_{2}=-\left(\partial_{n} \hat{u}\right) f_{2}+3 \alpha u_{0}\left(\partial_{n} u_{0}\right)-\partial_{n} \operatorname{div} \hat{u} \\
& \partial_{n} f_{2}=-3 \alpha\left(\partial_{n} \hat{u}\right) u_{0}
\end{aligned}
$$


or, excluding $f_{2}$,

$$
\partial_{1}^{n}\left(\left(\partial_{n} \hat{u}\right) u_{0}\right)=-\partial_{n}\left(\left(\partial_{n} \hat{u}\right)\left(\partial_{n} \hat{u}\right) u_{0}+u_{0}\left(\partial_{n} u_{0}\right)-\frac{1}{3 \alpha} \partial_{n} \operatorname{div} \hat{u}\right) .
$$

This relation represents a differential reduction for the system (9).

The limit $\alpha \rightarrow 0$ of the reduced hierarchy corresponds to the volumepreserving case $(\mathrm{k}=0)$, while the limit $\alpha \rightarrow \infty$ corresponds to the hierarchy characterized by the relation $\left(\Psi^{0}\right)_{-}^{3}=0$ (Gelfand-Dikii reduction).

\section{$\mathrm{N}=2$. Systems connected with Plebanski second heavenly equation}

We will consider in more detail the construction of reductions in the case $N=2$, which contains Plebanski second heavenly equation and its generalization proposed by Dunajski [7].

$\mathrm{k}=\mathbf{0}$

Volume-preserving reduction (in this case it is area-preserving) corresponds to Plebanski generalization of the second heavenly equation [10]. Vector fields in the Lax-Sato equations of the hierarchy (10) are Hamiltonian (twodimensional divergence-free), and it is possible to write the reduced system (9) in terms of the potential $\Theta, u_{1}=\Theta_{y}, u_{2}=-\Theta_{x}, x=x^{1}, y=x^{2}$. After the identification $z=-t_{1}^{1}, w=t_{1}^{2}, \phi=u_{0}$ we get the Dunajski system [7]

$$
\begin{aligned}
& \Theta_{w x}+\Theta_{z y}+\Theta_{x x} \Theta_{y y}-\Theta_{x y}^{2}=\phi, \\
& \phi_{x w}+\phi_{y z}+\Theta_{y y} \phi_{x x}+\Theta_{x x} \phi_{y y}-2 \Theta_{x y} \phi_{x y}=0 .
\end{aligned}
$$

The hierarchy connected with this system is studied in detail in [10. The general system (9) in this notations reads

$$
\begin{aligned}
& \left(\partial_{z y}+\partial_{w x}\right) \hat{u}+\left[\partial_{y} \hat{u}, \partial_{x} \hat{u}\right]=\left(\partial_{y} \phi\right) \partial_{x}-\left(\partial_{x} \phi\right) \partial_{y}, \\
& \left(\partial_{z y}+\partial_{w x}+\left(\partial_{y} \hat{u}\right) \partial_{x}-\left(\partial_{x} \hat{u}\right) \partial_{y}\right) \phi=0
\end{aligned}
$$

where $\hat{u}=u_{1} \partial_{x}+u_{2} \partial_{y}$.

$\mathrm{k}=1$

The reduction with $k=1$ is characterized by the relation

$$
J_{0}=\exp \alpha\left(\Psi^{0}-\lambda\right) .
$$


Generating equation for the reduced hierarchy is

$$
\left(\exp \left(-\alpha \Psi^{0}\right) \mathrm{d} \Psi^{0} \wedge \mathrm{d} \Psi^{1} \wedge \mathrm{d} \Psi^{2}\right)_{-}=0 .
$$

The reduction implies the existence of the solution $-\alpha \lambda$ of nonhomogeneous linear equations (12). The reduction condition for equations (23) is

$$
\operatorname{div} \hat{u}:=\partial_{x} u_{1}+\partial_{y} u_{2}=\alpha \phi .
$$

Using this relation, it is possible to exclude the function $u_{0}$ from the system (23) and obtain the reduced system in explicit form,

$$
\left(\partial_{z y}+\partial_{w x}\right) \hat{u}+\left[\partial_{y} \hat{u}, \partial_{x} \hat{u}\right]=\alpha^{-1}\left(\left(\partial_{y} \operatorname{div} \hat{u}\right) \partial_{x}-\left(\partial_{x} \operatorname{div} \hat{u}\right) \partial_{y}\right)
$$

The limit $\alpha \rightarrow 0$ of the reduced hierarchy corresponds to the Dunajski system hierarchy, while the limit $\alpha \rightarrow \infty$ corresponds to the hierarchy characterized by the relation $\Psi^{0}=l[10,18]$. For this hierarchy vector fields of Lax-Sato equations (7) do not contain a derivative with respect to a spectral variable, and $\phi$ in equations (23) is equal to zero,

$$
\left(\partial_{z y}+\partial_{w x}\right) \hat{u}+\left[\partial_{y} \hat{u}, \partial_{x} \hat{u}\right]=0
$$

This hierarchy is a 'precursor' of Plebanski second heavenly equation hierarchy [19, 18] corresponding to Hamiltonian vector fiels both in Lax-Sato equations (7) and in equation (24), which reduces to Plebanski second heavenly equation

$$
\Theta_{w x}+\Theta_{z y}+\Theta_{x x} \Theta_{y y}-\Theta_{x y}^{2}=0 .
$$

Thus the reduction (13) with $k=1$ is 'interpolating' between the hierarchy, connected with Dunajski system (22) and the hierarchy connected with the system (24), which for Hamiltonian vector fields reduces to Plebanski second heavenly equation.

$\mathrm{k}=\mathbf{2}$

Reduction with $k=2$ is characterized by the relation

$$
J_{0}=\exp \left(\alpha\left(\Psi^{0}\right)_{-}^{2}\right)
$$

Generating equation for the reduced hierarchy is

$$
\left(\exp \left(-\alpha\left(\Psi^{0}\right)^{2}\right) \mathrm{d} \Psi^{0} \wedge \mathrm{d} \Psi^{1} \wedge \mathrm{d} \Psi^{2}\right)_{-}=0 .
$$


Reduction conditions in terms of the system (23) are

$$
\begin{aligned}
& \partial_{z} \phi-\left(\partial_{x} \hat{u}\right) \phi-\frac{1}{2 \alpha} \partial_{x} \operatorname{div} \hat{u}=0, \\
& \partial_{w} \phi+\left(\partial_{y} \hat{u}\right) \phi+\frac{1}{2 \alpha} \partial_{y} \operatorname{div} \hat{u}=0 .
\end{aligned}
$$

The limit $\alpha \rightarrow 0$ corresponds to Dunajski system (22), and the limit $\alpha \rightarrow \infty$ - to the second Gelfand-Dikii reduction $\left(\Psi^{0}\right)_{-}^{2}=0$ for the system (24).

$\mathrm{k}=3$

Reduction with $k=3$ is characterized by the relation

$$
J_{0}=\exp \left(\alpha\left(\Psi^{0}\right)_{-}^{3}\right) .
$$

Generating equation for the reduced hierarchy is

$$
\left(\exp \left(-\alpha\left(\Psi^{0}\right)^{3}\right) \mathrm{d} \Psi^{0} \wedge \mathrm{d} \Psi^{1} \wedge \mathrm{d} \Psi^{2}\right)_{-}=0 .
$$

Reduction conditions in terms of the system (23) are

$$
\begin{aligned}
& \partial_{z}\left(\left(\partial_{x} \hat{u}\right) \phi\right)=\partial_{x}\left(\left(\partial_{x} \hat{u}\right)\left(\partial_{x} \hat{u}\right) \phi+\phi\left(\partial_{x} \phi\right)-\frac{1}{3 \alpha} \partial_{x} \operatorname{div} \hat{u}\right) \\
& \partial_{w}\left(\left(\partial_{y} \hat{u}\right) \phi\right)=-\partial_{y}\left(\left(\partial_{y} \hat{u}\right)\left(\partial_{y} \hat{u}\right) \phi+\phi\left(\partial_{y} \phi\right)-\frac{1}{3 \alpha} \partial_{y} \operatorname{div} \hat{u}\right) .
\end{aligned}
$$

The limit $\alpha \rightarrow 0$ corresponds to Dunajski system (22), and the limit $\alpha \rightarrow \infty$ - to the third Gelfand-Dikii reduction $\left(\Psi^{0}\right)_{-}^{3}=0$ for the system (24).

\section{Characterization of reductions in terms of the dressing data}

A dressing scheme for the hierarchy (317) can be formulated in terms of $(\mathrm{N}+1)$-component nonlinear Riemann-Hilbert problem on the unit circle $S$ in the complex plane of the variable $l$,

$$
\begin{aligned}
& \Psi_{\text {in }}^{0}=F_{0}\left(\Psi_{\text {out }}^{0}, \Psi_{\text {out }}^{1}, \ldots, \Psi_{\text {out }}^{N}\right), \\
& \Psi_{\text {in }}^{k}=F_{k}\left(\Psi_{\text {out }}^{0}, \Psi_{\text {out }}^{1}, \ldots, \Psi_{\text {out }}^{N}\right), \quad 1 \leqslant k \leqslant N,
\end{aligned}
$$

where the functions $\Psi_{\text {in }}^{0}(l, \mathbf{t}), \Psi_{\text {in }}^{k}(l, \mathbf{t})$ are analytic inside the unit circle, the functions $\Psi_{\text {out }}^{0}(l, \mathbf{t}), \Psi_{\text {out }}^{k}(p, \mathbf{t})$ are analytic outside the unit circle and 
have an expansion of the form (4), (5). The functions $F_{0}, F_{k}$ are suggested to define (at least locally) a diffeomorphism in $\mathbb{C}^{N+1}, \mathbf{F} \in \operatorname{Diff}(\mathrm{N}+1)$, and we call them the dressing data. In compact form the problem (25) can be written as

$$
\boldsymbol{\Psi}_{\text {in }}=\mathbf{F}\left(\boldsymbol{\Psi}_{\text {out }}\right) \text {. }
$$

It is straightforward to demonstrate that the problem (25) implies the analyticity of the differential form

$$
\Omega_{0}=J_{0}^{-1} \mathrm{~d} \Psi^{0} \wedge \mathrm{d} \Psi^{1} \wedge \cdots \wedge \mathrm{d} \Psi^{N}
$$

(where the independent variables of the differential include all the times $\mathbf{t}$ and $l$ ) in the complex plane and the generating relation (3), thus defining a solution of the hierarchy. Considering a reduction to the group of volumepreserving diffeomorphisms $\operatorname{SDiff}(\mathrm{N}+1)$, we obtain a reduction of the general hierarchy (3) to the case $J_{0}=1$ (divergence-free vector fields),

$$
\left(\mathrm{d} \Psi^{0} \wedge \mathrm{d} \Psi^{1} \wedge \cdots \wedge \mathrm{d} \Psi^{N}\right)_{-}=0 .
$$

For $N=2$ it is the Dunajski system hierarchy.

To construct a class of reductions (13), it is necessary to consider a kind of 'twisted' volume-preservation condition. Let the functions $G_{q}\left(y_{0}, \ldots, y_{n}\right)$, $0 \leqslant q \leqslant N$, define a volume-preserving diffeomorphism, $\mathbf{G} \in \operatorname{SDiff}(\mathrm{N}+1)$,

$$
\left|\frac{D\left(G_{0}, \ldots, G_{N}\right)}{D\left(y_{0}, \ldots, y_{N}\right)}\right|=1
$$

where for the Jacobian we use a notation

$$
\left|\frac{D\left(y_{1}, \ldots, y_{N}\right)}{D\left(x_{1}, \ldots, x_{N}\right)}\right|=\operatorname{det} \frac{D\left(y_{1}, \ldots, y_{N}\right)}{D\left(x_{1}, \ldots, x_{N}\right)}=\operatorname{det}\left(\frac{\partial y_{i}}{\partial x_{j}}\right) .
$$

Let us fix a set of analytic functions $f_{q}\left(y_{0}, \ldots, y_{n}\right)$ (the reduction data) defining a diffeomorphism and consider a problem

$$
\mathbf{f}\left(\boldsymbol{\Psi}_{\text {in }}\right)=\mathbf{G}\left(\mathbf{f}\left(\Psi_{\text {out }}\right)\right)
$$

which corresponds to the reduction of the hierarchy. The reduction condition for the dressing data of the problem (26) reads

$$
\mathbf{f} \circ \mathbf{F} \circ \mathbf{f}^{-1} \in \operatorname{SDiff}(\mathrm{N}+1) .
$$

This is what we call a 'twisted' volume-preservation condition. In terms of equations of the MS hierarchy the reduction is characterized by the relation

$$
\left(\mathrm{d} f_{0}(\boldsymbol{\Psi}) \wedge \cdots \wedge \mathrm{d} f_{N}(\mathbf{\Psi})\right)_{\text {in }}=\left(\mathrm{d} f_{0}(\Psi) \wedge \cdots \wedge \mathrm{d} f_{N}(\mathbf{\Psi})\right)_{\text {out }},
$$


thus the differential form

$$
\Omega_{\text {red }}=\mathrm{d} f_{0}(\Psi) \wedge \cdots \wedge \mathrm{d} f_{N}(\Psi)
$$

is analytic in the complex plane, and the reduced hierarchy is defined by the generating relation

$$
\left(\mathrm{d} f_{0}(\Psi) \wedge \cdots \wedge \mathrm{d} f_{N}(\Psi)\right)_{-}=0
$$

or, equivalently,

$$
\left(\left|\frac{D\left(f_{0}, \ldots, f_{N}\right)}{D\left(\Psi^{0}, \ldots, \Psi^{N}\right)}\right| \mathrm{d} \Psi^{0} \wedge \mathrm{d} \Psi^{1} \wedge \cdots \wedge \mathrm{d} \Psi^{N}\right)_{-}=0 .
$$

The diffeomorphism $\mathbf{f}$ for the reduced hierarchy is defined modulo a volumepreserving diffeomorphism. To obtain the reduced hierarchy (15), it is possible to use a symmetric choice

$$
\begin{aligned}
& f_{0}(\boldsymbol{\Psi})=\Psi^{0}, \\
& f_{n}(\boldsymbol{\Psi})=\exp \left(-\alpha N^{-1}\left(\Psi^{0}\right)^{k}\right) \Psi^{n}, \quad 1 \leqslant n \leqslant N .
\end{aligned}
$$

Thus we come to the following conclusion:

Proposition 3 In terms of the dressing data for the problem (26), a class of reductions (13) is characterized by the condition (28), where $\mathbf{f}$ is defined by (29).

\section{Acknowledgments}

The author is grateful to S.V. Manakov for useful discussions. This research was partially supported by the Russian Foundation for Basic Research under grants no. 09-01-92439, 10-01-00787 and by the President of Russia grant 4887.2008.2 (scientific schools). The participation in the conference "Nonlinear Physics. Theory and experiment. VI", where the talk resulting in this paper was presented, was made possible due to RFBR travel grant 10-01-08119 and the hospitality of the organizers.

\section{References}

[1] L. V. Bogdanov, On a class of reductions of the Manakov-Santini hierarchy connected with the interpolating system, J Phys. A: Math. Theor. 43 (2010) 115206 
[2] S. V. Manakov and P. M. Santini, The Cauchy problem on the plane for the dispersionless Kadomtsev-Petviashvili equation, JETP Lett. 83 (2006) 462-6.

[3] S. V. Manakov and P. M. Santini, A hierarchy of integrable PDEs in 2+1 dimensions associated with 2-dimensional vector fields, Theor. Math. Phys. 152 (2007) 1004-1011.

[4] S. V. Manakov and P. M. Santini, On the solutions of the dKP equation: the nonlinear, Riemann-Hilbert problem, longtime behaviour, implicit solutions and wave breaking, J Phys. A: Math. Theor. 41 (2008) 055204.

[5] Maciej Dunajski, An interpolating dispersionless integrable system, $J$. Phys. A: Math. Theor. 41 (2008) 315202

[6] M.V. Pavlov, Integrable hydrodynamic chains, J. Math. Phys. 44(9) (2003) 4134-4156

[7] M. Dunajski, A class of Einstein-Weyl spaces associated to an integrable system of hydrodynamic type, J. Geom. Phys. 51 (2004) 126-137.

[8] L. Martínez Alonso and A. B. Shabat, Energy-dependent potentials revisited: a universal hierarchy of hydrodynamic type, Phys. Lett. A 300 (2002) 58-64.

[9] L. Martínez Alonso and A. B. Shabat, Hydrodynamic reductions and solutions of a universal hierarchy, Theor. Math. Phys. 140 (2004) 10731085

[10] L. V. Bogdanov, V. S. Dryuma and S. V. Manakov, Dunajski generalization of the second heavenly equation: dressing method and the hierarchy, J Phys. A: Math. Theor. 40 (2007) 14383-14393

[11] L.V. Bogdanov, A class of multidimensional integrable hierarchies and their reductions, Theoretical and Mathematical Physics 160(1) (2009) $887-893$

[12] J.F. Plebański, J. Math. Phys. 16, 2395-2402 (1975)

[13] M.Dunajski, Proc.Roy.Soc.Lond.A 458, 1205 (2002)

[14] Jimbo M. and Miwa T. Solitons and infinite-dimensional Lie algebras. Publ. Res. Inst. Math. Sci. 19(3) (1983), 943-1001. 
[15] Zakharov V. E. Description of the $n$-orthogonal curvilinear coordinate systems and Hamiltonian integrable systems of hydrodynamic type. I. Integration of the Lamé equations, Duke Math. J. 94(1) (1998), 103139 .

[16] Zakharov V.E. and Manakov S.V. DAN, 360(3) (1998), 324-327.

[17] L.V. Bogdanov and E.V. Ferapontov, Projective differential geometry of higher reductions of the two-dimensional Dirac equation, Journal of Geometry and Physics 52(3) (2004) 328-352

[18] L.V. Bogdanov and B.G. Konopelchenko, On the $\bar{\partial}$-dressing method applicable to heavenly equation, Physics Letters A 345(1-3) (2005) 137143

[19] K. Takasaki, J. Math. Phys. 30(7), 1515-1521 (1989)

[20] K. Takasaki, J. Math. Phys. 31(8), 1877-1888 (1989) 